\title{
DIÁLOGO Y ESTILO EN LA CELESTINA
}

Este ensayo es parte del segundo capítulo de un libro que se titulará El arte de Fernando de Rojas. Como dejo sentado en el primer capítulo, parto del supuesto de que Rojas es autor de los actos añadidos y de las interpolaciones de 1502, según lo que él mismo afirma. Es éste un motivo de grandes discusiones entre los eruditos, pero si aceptamos la idea de que Rojas escribió tanto la Tragicomedia (la versión ampliada) como la Comedia (los dieciséis actos originales), hemos de admitir que debió hacerlo después de un largo período de meditación, y con una renovada conciencia de su arte. Los nuevos actos e interpolaciones son, en este sentido, como una "segunda parte" de la Celestina, una segunda parte comparable a la del Quijote. Y si leemos el libro de esta manera, podremos hasta cierto punto reconstruir la visión que Rojas tuvo de su obra. Descontando las adiciones más importantes, muchos cambios de menor monta nos sirven también como valiosas claves para descubrir la técnica creadora de Rojas. Nos imaginamos el furor crítico que se desataría si para una obra de Shakespeare se descubriese un texto igualmente rico en variantes. Y teniendo en cuenta que tanto la intenciôn de Rojas como su obra son tan difíciles de precisar - por ser auténticamente humanas- como las de Shakespeare, es evidente que los estudiosos de la literatura española han dejado escapar hasta ahora una inestimable oportunidad.

El lector se dará cuenta de que no comentamos la paternidad del primer Acto, otro tema de acaloradas discusiones, y, también, de que no tratamos de "probar" que un solo autor haya escrito el resto de la obra. Esto último es mera suposición preliminar (no podría ser de otra manera en este trabajo), que nos permitirá darnos cuenta de las preocupaciones estilísticas de Rojas. Si llegamos a algunas conclusiones nuevas sobre la paternidad de la obra (las interpolaciones que estudiamos no han merecido por lo común la atención de los críticos de la Tragicomedia), ello no será sino consecuencia accesoria de nuestras indagaciones sobre el arte del estilo en Rojas.

Ahora bien, el intento de redescubrir la Celestina desde el punto de vista del arte de Rojas nos obliga a definir la palabra "estilo". Para nuestro propósito, tiene un sentido más próximo a su antepasado stylus que los sentidos que sugieren Juan de Valdés o Leo 
Spitzer, para sólo mencionar a dos críticos que se preocupan por este aspecto de la obra de arte. "Estilo" significa aquí, necesariamente, el resultado a que conduce la labor crítica del artista en cuanto a sugerencia, aceptación o rechazo de cierta palabra en un texto. Tiene que ver con esas ideas de orden, propiedad y efecto agradable que guían la pluma de un artista literario. Es el producto de su preocupación consciente, aunque no forzosamente académica, por la palabra escrita, y si bien es cierto que las normas tácitas de una época o de una "escuela literaria" son importantes en toda definición de este tipo, damos por supuesto que la conciencia verbal de un autor individual es superior a todas las recetas. Esta manera de concebir el estilo como conciencia especial del lenguaje está a medio camino entre la concepción de Valdés y la de Spitzer (cada una de las cuales representa una idea de "estilo" posible y aceptada por muchos). Lo que preocupaba a Valdés era quizá, no tanto el arte del escritor, cuanto los efectos producidos por el "stilo" en el lector, efectos que dependían de su adopción de ciertos principios esenciales y aparentemente absolutos de buen gusto. Spitzer, por el contrario, relaciona tan íntimamente el estilo con su creador y su época, que prefiere subrayar las posibilidades de que el estilo traicione inconscientemente a ambos, traición de que el crítico puede sacar un excelente partido. Pero, para este trabajo, ni el lector ni el escritor bastan por sí solos; el arte estilístico de Rojas se funda en el criterio, a la vez individual e impersonal, que oración tras oración va rigiendo el lenguaje de la Celestina.

El proponer una definición tan elemental de "estilo" no quiere decir que rechacemos arbitrariamente el punto de vista de Spitzer o el de Juan de Valdés. Corresponde más bien, según hemos dicho, a nuestro modo especial de ver la Celestina, al hecho de que la preocupación por las palabras es en Rojas el aspecto más visible de su arte. Mucho de lo que vamos a decir se funda en los métodos actuales de análisis estilístico, y al mismo tiempo debemos admitir que la conciencia del lenguaje, en Rojas, dependía seguramente de un concepto de estilo que ya apuntaba hacia el que habría de aceptarse cincuenta años más tarde. Admitimos la validez y la necesidad de ambos puntos de vista, pero preferimos, por lo menos en este caso, dejar a un lado el juicio estético y la elaboración de la intuición. Ninguna de estas dos cosas nos permite reconstruir la consciente preocupación por la palabra en la obra de Rojas, preocupación que salta a la vista en las interpolaciones y supresiones de 1502. Rojas, uno de los artistas más atentos al fenómeno de la conciencia humana, parece haber tenido (por lo menos cuando corrigió la Comedia) clara conciencia de la naturaleza de su propio arte. Así, una de las maneras de conocer los recursos personales del estilo 
de Rojas puede basarse en un estudio de las palabras de la versión corregida.

Inútil es decir que las interpolaciones, por su sola presencia, indican que Rojas creía necesario cambiar los pasajes en que aparecen. En este sentido, todas revelan virtualmente su arte del estilo. No sólo añaden, sino que corrigen, y es interesante comparar el estilo del pasaje corregido con el del contexto original. Pero, al considerar las interpolaciones en su conjunto, resulta que, desde el punto de vista de Rojas, pueden dividirse en dos clases, una de las cuales se relaciona más íntimamente con los problemas del estilo que la otra. $\mathrm{Si}$, como hemos dicho, la reelaboración fué fruto de una larga meditación sobre lo ya creado, es evidente que algunos cambios reflejan la nueva visión que Rojas tenía de toda la obra, de su unidad temática, de su división en actos, de la evolución de sus personajes; otros, en cambio, parecen corresponder a una lectura cuidadosa de las partes menores de la obra; la palabra, la oración, el párrafo o, a lo sumo, el parlamento. Estas últimas correcciones son las que mejor revelan el sentido que Rojas tiene del estilo, y la confrontación con el texto original nos muestra de qué modo fué plasmando el lenguaje. El primer tipo de interpolaciones nos hace ver una conciencia de las líneas creadoras por encima del nivel de las palabras; el otro, la disposición de las palabras mismas ${ }^{1}$.

Si estudiamos todas las interpolaciones de una o dos palabras (interpolaciones que, casi por definición, son de orden estilístico), veremos que hay un grupo que resalta sobre los demás: viento?

GEL.-¿Qué pensauas, Sempronio? ¿Auíame de mantener del

Cen..-iPara adalid eres tú bueno, cargado de agüeros $\mathrm{e}$ recelo!

Cet.- ;O mis enamorados, mis perlas de oro! ¡Tal me venga el año qual me parece vuestra venida!

${ }^{1}$ Naturalmente, estos dos tipos de interpolaciones no son del todo independientes uno de otro. Por ejemplo, muchas de las interpolaciones que pretenden reforzar el orden del material temático reflejan asimismo una preocupación por la circunstancia lingüística. Sin embargo, la distinción es útil para nuestro propósito: nos permite dar nueva importancia a una serie de modificaciones del texto de la Celestina que han pasado por alto o han interpretado mal los críticos interesados únicamente en encontrar apoyos para sus teorias acerca de la paternidad de la obra. Entre las correcciones principalmente estilísticas, según nuestra definición, encontramos no sólo adiciones de proverbios y de pasajes eruditos, sino lo que hemos llamado "autocorrecciones mínimas", supresiones o inserciones de una o dos palabras no más. Empezaremos con estas correcciones; mediante su estudio esperamos encontrar una relación más íntima entre la palabra y el arte del estilo. 
Melib.-Amiga Lucrecia e mi leal criada $e$ fiel secretaria, ya has visto cómo no ha sido más en mi mano.

Cal.-Descuelga, Pármeno, mis coraças, e armáos vosotros, $e$ assi yremos a buen recaudo, porque, como dizen: el hombre apercebido, medio combatido.

Sos.-Señor, vna muger que se llamaua Celestina ${ }^{2}$.

Es evidente en estas pequeñas interpolaciones, hasta ahora poco notadas, la conciencia que tiene Rojas de los requisitos estilísticos fundamentales del diálogo, la preocupación de que cada parlamento vaya adecuadamente dirigido a la segunda persona, de que exista en función del hablante y del oyente y no sólo para instrucción o deleite del lector. Las palabras añadidas ponen de relieve la intencionalidad interna del lenguaje. Es un lenguaje hablado (aunque no siempre popular), en cuanto que está escrito como si saliera de una vida y se dirigiera hacia otra. Cada palabra se sostiene en un yo y un $t \dot{u}$ y da acceso a un yo y un $t u ́$. El diálogo, para Rojas, es el lenguaje que resulta del encuentro de dos vidas.

Esta manera de gobernar el estilo por los requisitos del diálogo vivo es tan importante en el arte de Rojas, que merece ser ejemplificada en detalle. Examinemos dos de las interpolaciones citadas para darnos cuenta de su valor real y ver cómo no son meras adiciones mecánicas o superficiales puestas con el fin de dar la apariencia de diálogo. El contexto de la primera interpolación es el siguiente (Acto III, vol. I, pág. 133):

Semp.-Haz a tu voluntad, que no será éste el primer negocio que has tomado a cargo.

CEL.-¿El primero, hijo? Pocas vírgines, a Dios gracias, has tú visto en esta cibdad, que hayan abierto tienda a vender, de quien yo no aya sido corredora de su primer hilado. En nasciendo la mochacha, la hago escriuir en mi registro, e esto para saber quántas se me salen de la red. ¿Qué pensauas, Sempronio? ¿Auíame de mantener del viento? ¿Heredé otra herencia? ¿Tengo otra casa o viña? ¿Conócesme otra hazienda más deste oficio? ¿De qué como e beuo? ¿De qué visto e calço? En esta cibdad nascida, en ella criada, manteniendo honrra, como todo el mundo sabe, ¿conoscida, pues, no soy? Quien no supiere mi nombre e mi casa tenle por estranjero.

Este parlamento está hecho evidentemente de dos partes, una indicativa (narración jactanciosa) y otra interrogativa (serie de preguntas

2 Las interpolaciones van en cursiva. Citamos por la edición de Cejador, Madrid, 1913. Los seis pasajes corresponden a: Acto III (vol. I, pág. 133); Acto III (vol. I, pág. 140); Acto IX (vol. II, pág. 27) ; Acto X (vol. II, págs. 66-67); Acto XII (vol. II, pág. 83); y Acto XIII (vol. II, pág. 118). 
más o menos retóricas, que no esperan respuesta). La primera comienza con palabras enderezadas al oyente - ¿El primero, hijo?"-, pero esto apenas es suficiente para mantener la atención de Sempronio, que es quien ha de ser impresionado por el discurso; así, la primera pregunta de la segunda parte - ¿Qué pensauas?”-, por retórica que sea, va enderezada directamente a él, con lo cual se le hace blanco de las preguntas que siguen. Pero en 1502 Rojas no estaba todavía satisfecho y, en este momento estratégico, quiso aumentar la autenticidad del discurso como diálogo. "¿Qué pensauas, Sempronio?" es, en efecto, estilísticamente necesario no sólo para Rojas, sino para Celestina misma, y casi podemos palpar la falta del nombre en la Comedia.

Más necesario aún para la fuerza expresiva del diálogo es quizá el " $t u$ '" del segundo ejemplo. Sempronio ha seguido dudando de la capacidad profesional de Celestina y le recuerda, indirectamente, un fracaso anterior por cuya causa fué emplumada. Celestina responde con enojo:

¿Alahé, en malora a ti he yo menester para compañero! iAvn si quisieses auisar a Celestina en su oficio! Pues quando tú nasciste ya comía yo pan con corteza. ¡Para adalid eres tú bueno, cargado de agüeros e recelo!

Poco hay que explicar aquí. Los pronombres yo y tú son fundamentales para la expresión, en forma de diálogo, de la violenta reacción de Celestina, de su espontánea réplica no tanto a las dudas de Sempronio cuanto al insulto implícito. Y Rojas subraya la oposición afectiva entre primera y segunda personas al añadir el " $t u$ " de la última oración; termina el parlamento poniendo de relieve la indignación -indignación en forma de diálogo- de Celestina. En uno y otro caso es evidente que la interpolación no es una segunda mano de barniz que se pasa sobre el diálogo, sino que refuerza un rasgo que ya existía antes: ese apuntar a la segunda persona. En ambos casos el yo del hablante no sólo se expresa a sí mismo, sino que se proyecta sobre el tu del oyente, y Rojas, al corregir su propio texto, hace resaltar la importancia que este requisito del diálogo tiene en su arte del estilo.

Lo que acabamos de señalar no es, desde luego, una cualidad privativa de la Celestina. Cualquier gran novelista, dramaturgo o poeta épico conoce intuitivamente las relaciones entre el estilo y el diálogo. Pero quizá ninguno pueda compararse con Rojas en el consciente y concienzudo desarrollo de un estilo basado en esta relación; y hasta ahora no se ha atendido debidamente a la gran sutileza de su diálogo. Podemos escoger al azar un pasaje de la obra (con excepción del primer Acto y de los frecuentes monólogos), y observaremos de inmediato la mutua presencia de un yo y un tú que deciden la ordenación de las palabras. La palabra es, en la Celestina, un puente entre el hablante y el oyente, el punto de reunión de dos vidas. El ejemplo 
que sigue (Acto IV, vol. I, págs. 191-192) es característico. Celestina ha estado hablando aparte con Lucrecia, al fin de su primera entrevista con Melibea:

Melib.-¿Qué le dizes, madre?

CeL.-Señora, acá nos entendemos.

Meisb.-Dímelo, que me enojo quando yo presente se habla cosa de que no aya parte.

Cen.-Señora, que te acuerde la oración para que la mandes escriuir, e que aprenda de mí a tener mesura en el tiempo de tu yra, en la qual yo vsé lo que se dize: que del ayrado es de apartar por poco tiempo, del enemigo por mucho. Pues tú, señora, tenías yra con lo que sospechaste de mis palabras, no enemistad; porque, avnque fueran las que tú pensauas, en sí no eran malas, que cada día ay hombres penados por mugeres e mugeres por hombres, e esto obra la natura, e la natura ordenóla Dios, e Dios no hizo cosa mala. E assí quedaua mi demanda, como quiera que fuesse, en sí loable, pues de tal tronco procede, e yo libre de pena...

Al llegar a este punto, Melibea ya no puede dominar a Celestina, aunque la amenaza débilmente con un nuevo enojo: estas quejas femeninas son, en efecto, señal de derrota y preparan el terreno para la respuesta de Celestina. Y ésta, al responder, en total dominio de la situación, prepara su siguiente ofensiva. Al principio, da una explicación endeble de su aparte con Lucrecia - "que te acuerde la oración ..."-, y luego, sin dar tiempo a que Melibea ponga en duda la explicación, le resume la entrevista. Lo hace empleando las palabras precisas que quisiera dejar grabadas en el espíritu de su oyente, preparando hábilmente las razones que siguen: 'no eres mi enemiga; estabas airada, pero sólo de manera momentánea'. Luego, con mayor atrevimiento: 'aunque lo que te irritó fuera cierto, si lo ves bien no tenía por qué haber sido necesariamente malo'. Y por fin insinúa que las sospechas de Melibea eran quizá justificadas, aunque no se atreve todavía a confirmar la insinuación: “... mi demanda, como quiera que fuesse".

Pero volvamos al estilo. Tenemos aquí, en apariencia, el lenguaje típico de todo argumento objetivo y racional, de toda afirmación de tipo general que se aplica a un caso particular. Viéndolo más de cerca, sin embargo, notamos una serie de transiciones aparentemente irracionales. El $e$ que de la primera oración de Celestina, con su introducción de "mesura" y la distinción entre ira y enemistad, no corresponde a nada de lo que Celestina decía en secreto a Lucrecia. El porque con que se pasa a la tercera oración es también ambiguo: no hay ninguna relación causal entre la airada reacción de Melibea al sospechar lo que quería decir Celestina, y el esfuerzo de ésta por legitimar sus palabras, "avnque fueran las que tú pensauas". Así, la 
argumentación lógica no es más que una máscara; y las palabras escogidas responden verdaderamente a la presencia viva de Melibea como oyente. En efecto, cada palabra está calculada para provocar una reacción vital (aunque, al mismo tiempo, puede traicionar los sentimientos de quien habla), y, como resultado, la auténtica estructura del pasaje citado no es propiamente la lógica, sino la vital alternancia de la primera y la segunda personas. Fijémonos en estas pala bras: "Señora, que te acuerde..."; "que aprenda de $m i$ a tener mesura en el tiempo de $t u$ yra, en la qual yo vsé..."; "Pues $t u$, señora, tenias yra..."; "avnque fueran las que tú pensauas..."; "e yo libre de pena". Esta abundancia de primeras y segundas personas indica una subordinación de lo general - "del ayrado es de apartar por poco tiempo", o "Dios no hizo cosa mala"- a la confrontación de dos vidas. El arte estilístico de Rojas es, ante todo, un arte de diálogo vital.

Tanto es así, que podríamos aplicar a todo el estilo una de las observaciones más agudas que se hacen sobre Celestina (observación interpolada en la edición de 1502): "Lo que en sus cuentas reza es ... qué despenseros le dan ración e quál lo mejor e cómo les llaman por nombre, por que quando los encontrare no hable como estraña" (Acto IX, vol. II, pág. 26); y Sempronio continúa: "Quando menea los labios es fengir mentiras, ordenar cautelas para hauer dinero: por aquí le entraré, esto me responderá, estotro replicaré. Assí viue ésta ..." En efecto, la vida de Celestina, como todas las vidas de la Tragicomedia, está dedicada al diálogo. Son tantas las interpolaciones que confirman la importancia de este elemento en el estilo de Rojas, que sería imposible enumerarlas todas; pero no podemos resistir a la tentación de mencionar la primera interpolación del Acto IV (vol. I, pág. 163); Alisa ha anunciado que tiene que dejar sola a Melibea con Celestina, y entonces ésta exclama, en aparte:

Por aquí anda el diablo aparejando oportunidad, arreziando el mal a la otra. iEa, buen amigo! iTener rezio! Agora es mi tiempo o nunca. No la dexes, lléuamela de aqui a quien digo.

En la Comedia de dieciséis actos, este aparte (y el que en el mismo acto, más adelante, precede a "iCe, hermano, que se va todo a perder!") no pasaba de ser una referencia a la solemne invocación con que termina el Acto III. Pero la interpolación, con ese dirigirse íntimamente a un interlocutor, transmite la sensación que tiene Celestina de una presencia diabólica invisible. Su fe en lo sobrenatural emerge del reino de fórmulas y conjuros y se aviva al soplo de la esperanza y de la desesperación de estos dos momentos. Ya no se refiere Celestina al "triste Plutón": ahora que las circunstancias han dado una dirección a su superstición, habla con su "buen amigo" y "hermano”. ¡Cuánto más vigoroso es este murmurar que el de la Comedia 
original, y, al mismo tiempo, cómo concuerda con los rasgos más personales del estilo de Rojas!

Enfocando de este modo las intenciones estilísticas del autor de la Celestina, podemos llegar a una nueva interpretación de otras características interesantes de su lenguaje y de su técnica. En primer lugar, la extraordinaria abundancia de preguntas. Más de la mitad de las oraciones llevan signo de interrogación, y no sería difícil que la $\mathrm{Ce}$ lestina fuera, relativamente, la obra de la literatura occidental en que más abunden las construcciones de este tipo ${ }^{3}$. Desde luego, las interrogaciones pocas veces pretenden lograr una respuesta informativa por parte del oyente. Pero, como vimos en la primera interpolación, cada pregunta invoca la presencia de un oyente. Así como las frecuentes exclamaciones expresan los sentimientos del yo, así todas estas preguntas suponen en lo que se dice la presencia del $t u$. El lenguaje casi nunca tiene vida propia fuera de la conversación, del diálogo de dos personas frente a frente.

Este mismo gobierno artístico se extiende naturalmente más allá del nivel del estilo y del lenguaje, y adopta muchas otras formas. Pensemos, por ejemplo, en la constancia con que aparece el tópico de los afeites femeninos a lo largo de la obra. El tópico era frecuente en la sátira social contemporánea y anterior -el Corbacho del Arcipreste de Talavera-, y Jorge Manrique alude brevemente a él para expresar la fugacidad de la vida; pero en la Celestina parece responder a necesidades distintas. Los afeites representan una especie de diálogo plástico, un diálogo visual que acompaña al hablado. Como dice Areusa al criticar a Melibea (Acto IX, vol. II, pág. 33), "Por vna vez que aya de salir donde pueda ser vista, enuiste su cara con hiel e miel, con vnas tostadas e higos passados e con otras cosas ..." En uno de los actos añadidos es evidente la misma preocupación: Elicia decide quitarse el luto por Celestina (Acto XVII, vol. II, pág. 167):

Mas para esto es el buen seso, viendo la pérdida al ojo, viendo que los atauíos hazen la muger hermosa avnque no lo sea, tornan de vieja moça e a la moça más. No es otra cosa la color e aluayalde sino pegajosa liga en que se trauan los hombres. Ande, pues, mi espejo e alcohol, que tengo dañados estos ojos; anden mis tocas blancas, mis gorgueras labradas, mis ropas de plazer. Quiero adereçar lexía para estos cabellos, que perdían ya la ruuia color, y esto hecho, contaré mis gallinas, haré mi cama, porque la limpieza alegra el coraçón, barreré mi puerta e regaré la calle, por que los que passaren vean que es ya desterrado el dolor.

${ }^{3}$ En su tesis doctoral para la Universidad de Columbia, Stantey M. SAPon (A study of the development of the interrogative in Spanish, Columbus, Ohio, 1951 ) calcula un porcentaje de 54.69 construcciones interrogativas en la Celes. tina, porcentaje correspondiente a $73^{6}$ preguntas. 
Los afeites, como la ropa, tienen aquí su razón de ser en función de los encuentros personales; por eso reaparecen incesantemente en la Celestina, no como lugar común, sino como preocupación vital.

Por último, y brevemente, podemos llamar la atención sobre el "diálogo" constante de ademanes y gestos a lo largo de la obra. Cada personaje está siemṕpre alerta al aspecto físico de los demás, no sólo a ese aspecto preparado artificiosamente por medio de afeites, sino a las expresiones físicas accidentales que revelan al interlocutor en la intimidad de su vida. Si los afeites se dirigen al $t \dot{u}$, los ademanes emergen del yo como otras tantas exclamaciones. Aunque Rojas deja en la sombra los rostros y sus matices de expresión (limitación que no hemos de explicar aquí), los cambios de actitud de Celestina, los saltos de desahogo de Pármeno al escapar del peligro (Acto XII), los arrobamientos de Calisto y muchos otros gestos expresivos sirven de complemento al diálogo de las palabras. El estilo, los tópicos y las acciones vienen a prestar consciente servicio a la primera y segunda personas que constituyen el paradigma artístico de Rojas.

Ohio State University.

Stephen Gilman 